\title{
A WELL-DOCUMENTED CASE OF CHRONIC RENAL FAILURE DUE TO MISPLACEMENT OF THE TRANSPLANTED KIDNEY
}

Paulo Rodrigues ${ }^{\mathrm{a}}$, Flavio Hering ${ }^{\mathrm{a}}$, Antonio Gil ${ }^{\mathrm{b}}$

\section{INTRODUCTION}

There may be many reasons for the malfunctioning of transplanted organs. Percutaneous biopsy can easily be undertaken to give greater specificity to possible immunological causes of rejection. ${ }^{1,2}$

Acute venous or arterial thrombosis frequently leads to graft loss after a short period of anuria which justifies a diagnosis of vascular occlusion. This causes graft dysfunction or loss in $3.5 \%$ to $12.5 \%$ of cases. ${ }^{3,4}$ Progressive malperfusion with rising or stabilized serum creatinine at higher levels demands aggressive imaging investigation to exclude possible aneurismal formation, progressive stenosis, or vascular fistula. This paper reports a rare case of kinking of the main artery due to organ rotation occurring after a simultaneous pancreas-kidney transplant.

\section{CASE REPORT}

A 52-year-old non-dialytic man with long-standing diabetes was submitted to simultaneous cadaveric pancreaskidney transplant through peritoneal access. The right kidney and the pancreas from the same donor were sewn into the left flank by venous and arterial termino-lateral anastomosis in the iliac vessels. After an uneventful operation, the serum creatinine level dropped from $4.5 \mathrm{mg} / \mathrm{dl}$ and stabilized at $1.8 \mathrm{mg} / \mathrm{dl}$ on the seventh PO day in conjunction with a mild hypertension. Magnetic resonance angiography (MRA) showed a well perfused kidney (Figure 1). The patient had a renal biopsy revealing acute tubular necrosis (ATN). Cyclosporine, prednisone and mycophenolate mofetil were maintained. On clinical follow-up, creatinine level varied from 2.2 to $4.4 \mathrm{mg} / \mathrm{dl}$ and a new biopsy revealed persistent ATN. The cyclosporine level was kept at $150-250 \mathrm{mg} / \mathrm{dl}$. After three months, new MRA imaging revealed a well perfused, but dystopic kidney (Figure 2) dis-

a Urology Department, Hospital Beneficência Portuguesa - São Paulo/SP, Brazil.

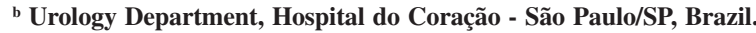
paulortrodrigues@uol.com.br

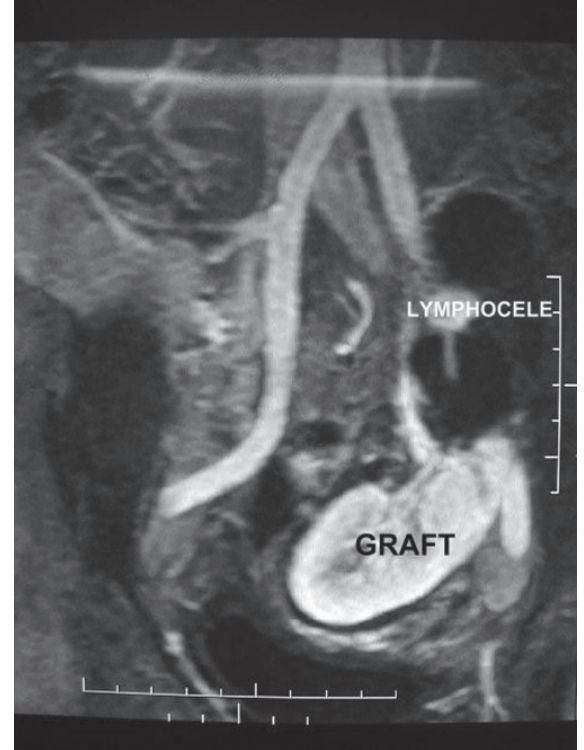

Figure 1 - Magnetic resonance showing an upper pole lymphocele displacing the renal graft.

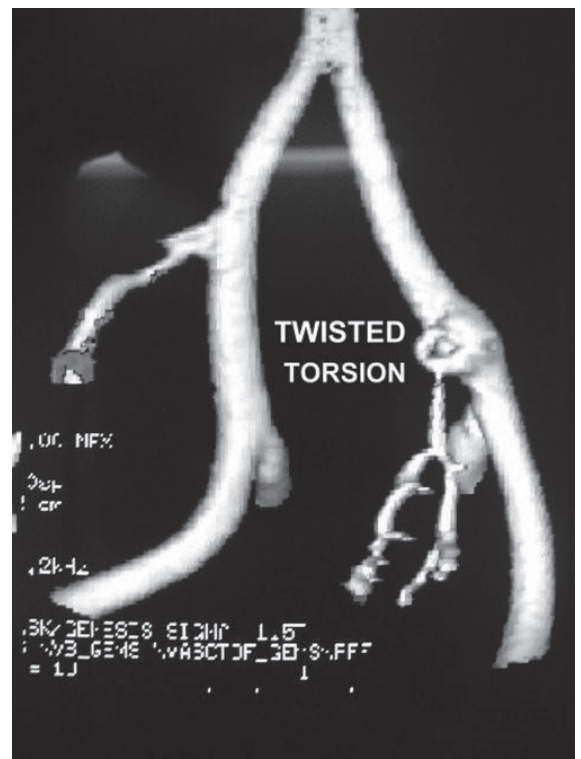

Figure 2 - Magnetic resonance angiography showing the main renal artery of the graft twisted. 
placed by a $500 \mathrm{ml}$ lymphocele in the upper pole.

On the $120^{\text {th }}$ PO day, the patient was submitted to open surgical exploration but the arterial de-torsion was quite difficult due to an intense fibrotic reaction, resulting in kidney retrieval after massive arterial lesion.

\section{DISCUSSION}

Complications arising from kidney transplants may result from immunological, urological, or vascular causes. ${ }^{5}$ Leakage of urine through the incision and/or persistent anuria are signs of early urological problems. These scenarios are usually investigated by percutaneous biopsy and ultrasound exploration, either of which may indicate problems with causes ranging from immunological to surgical issues.

Asymptomatic lymphocele collections are a well-known complication related to rejection or to improper donor lymphatic ligation during the harvesting dissection.

Lymphocele formation is a frequent event in kidney transplantation, occurring in $22 \%$ of cases, although it does not seem to be associated with the source of the kidney, but rather with rejections or ATN episodes. ${ }^{6}$

Most lymphocele formations are asymptomatic and do not harm the graft, but large or irregularly positioned formations may hamper the functioning of the ureter or impede vascular flow.

In our case, it is noteworthy that the decrease in the creatinine level ceased after the patient resumed walking, which possibly contributed to kidney displacement and torsion. Since fluid collection is more frequent in simultaneous pancreas-kidney grafting, some authors advocate intra-peritoneal placement of both organs in order to avoid retroperitoneal collections frequently seen from minor pancreatic collections.

The potential complication of renal pedicle torsion related to this approach is a concern, though it has never been reported in the literature. ${ }^{7}$ The kidney becomes loose in the peritoneal cavity and, as seen in our case, the intra-peritoneal approach did not prevent lymphocele formation.

Although widely discussed, torsion or compression of the pedicle and displacement of the graft have rarely been reported in the literature ${ }^{8,9}$ due to inadequate methods of visualization or restricted usage of imaging tools because of their invasiveness and lack of contrast. Arterial stenosis or kinking at the vascular anastomosis may occur in $3.5 \%$ to $12.5 \%$ of grafts, but invasive investigation could not be justified unless hypertension or progressive graft loss supervenes.

The advent of MRA has allowed for the study of the vascular pedicle with minimal clinical manipulation and no nephro-toxicity. Conventional arteriography, despite high resolution, is limited by its planar nature. MRA is a desirable approach due to the possibility of image reconstruction into a $3 \mathrm{D}$ image.

In our case, there was evident torsion of the pedicle in the post-operative period leading to stabilization of the decreasing creatinine levels at an elevated level with fluctuations thereafter. The torsion was evident in the open field, but the fibrosis impeded a simple manual de-torsion. Knotty resection and re-anastomosis led to thrombosis and graft loss, revealing the difficulty in approaching the pedicle in surgical revisions for vascular complications. As reported by others, this results in a higher rate of transplant nephrectomies. $^{3}$

It is impossible to determine the real cause of the described displacement, but it may be related to the intra-peritoneal position of the kidney frequently used for simultaneous pancreas-kidney transplants. This approach, as well as peritoneal fenestration, has been advised ${ }^{10}$ in order to minimize the formation of lymphoceles and to facilitate peritoneal absorption of intra-abdominal collections, but it did not provide absolute prevention in our case.

\section{REFERENCES}

1. Doyle AJ, Gregory MC, Terreros DA: Percutaneous renal biopsy: comparison of a $1.2 \mathrm{~mm}$ spring-driven system with a traditional $2 \mathrm{~mm}$ hard-driven system. Am J Kidney Dis. 1994;23:498-451.

2. Burstein DM, Korbet SM, Schartz MM: The use of the automatic core biopsy system in percutaneous renal biopsies: a comparative study. Am J Kidney Dis. 1993;22:545-548.

3. Roye SFS, van der Vliet JA, Hoitsma AJ, Reinaerts HHM, Buskens FGM: Early vascular complications of renal transplanation. Clin transplantation. 1993;7:496-500.

4. Rijksen JFW, Koolen MI, Warassewski JE: Vascular complications in 400 consecutive renal alotransplants. J Cardiovasc Surg. 1982;23:91-95.

5. Shoskes DA, Hanbury D, Cranston D, Morris PJ: Urological complications in 1000 consecutive renal transplant recipients. J Urol. 1995; 153:18-21.
6. Hauli RB, Stoff JS, Lovewell T: Post-transplant lymphoceles: a critical look into risk factors, pathophysiology and management. J Urol. 1993;150, 22-26.

7. Knight RJ, Matsumoto C: Renal pedicle torsion as a cause of acute allograft after kidney-pancreas transplantation. Abstract P1-09, Acta Chirurgica Astriaca, 8th Congress IPITA, Innsbruck. June 12-15, 2001, 33:174.

8. Williams SG,McVicar JP,Low RK: Endopyelotomy of ureteropelvic junction obstruction caused by torsion of a renal allograf. J Urol. 1999, 161:1560-1564.

9. Marvin RG, Halff GA, Elshihabi I: Renal allograft torsion associated with Prune-Belly syndrome. Ped Nephrol. 1995;9:8-10.

10. Zaontz MR, Firlit CF: Pelvic lymphocele after pediatric renal transplanation: A successful technique for prevention. J Urol. 1988;139:557-560. 